$2018,19(4)$, pp. 831 - 843, DOI: 10.18038/aubtda.405179

\title{
LONGITUDINAL STABILITY ANALYSIS OF A UAV UNDER THE UNCERTAINTY OF TWO STABILITY DERIVATIVES
}

\author{
Uğur ÖZDEMIIR ${ }^{1, *}$, Mehmet Serif KAVSAOĞLU ${ }^{1}$, Zafer ÖZNALBANT ${ }^{2}$, Ünver KAYNAK ${ }^{1}$ \\ ${ }^{1}$ Faculty of Aeronautics and Astronautics, Eskişehir Technical University, Eskişehir, Turkey \\ ${ }^{2}$ Turkish Aerospace Industries, Ankara, Turkey
}

\begin{abstract}
The longitudinal stability of an aircraft is analyzed using root locations of the transfer function's denominator (the characteristic equation). This transfer function is obtained by linearizing aircraft dynamic model at a certain operation point (altitude and speed). However, aircraft have varying stability derivatives, therefore dynamic behavior, for different flight phases such as takeoff, cruise, and landing. Thus the stability analysis of the characteristic equation can be said to be valid only for a single flight condition. In fact, stability derivatives vary with flight conditions, so an analysis that includes all possible stability derivatives in the flight envelope is required to guarantee stability. In this study, the two most variable stability derivatives in the transfer function were taken as uncertain parameters. Gridding these two parameters to check the stability of the unmanned aerial vehicle for all possible flight conditions is a possible method, but it is very time-consuming, and it cannot assure the stability theoretically. A new simple approach is developed by using the Edge and Bialas theorems, which guarantees stability despite the uncertainty of two stability derivatives. The problem is reduced to the analysis of four polynomials. With the eigenvalues of just four polynomials, the stability characteristics of an airplane for a given flight envelope can be easily determined.
\end{abstract}

Keywords: Stability analysis, Edge theorem, Bialas theorem, Unmanned aerial vehicles

\section{INTRODUCTION}

The $\mathrm{H}_{\infty}$ technique can adequately handle robust stability and performance issues under unstructured perturbations. Nevertheless, it is incomplete in the case of parameter uncertainty. Italian mathematician Faedo (1953) and Russian scientist Kharitonov (1970) introduced the treatment of robust stability problems under parameter uncertainty. An "optimal" feedback compensator for the system is obtained by the $\mathrm{H}_{2}$ and $\mathrm{H}_{\infty}$ methods. Before such a compensator can be formed, for the real-world applications, it is normal to demonstrate its abilities according to additional design criteria, not only optimal ones. Performance with parameter uncertainty is especially important for most systems, but optimal $\mathrm{H}_{2}$ and $\mathrm{H}_{\infty}$ method are unable to supply a direct and nonconservative solution to this significant problem. The problem of robustness under parameter uncertainty was the focus of Kharitonov's Theorem for interval polynomials. This Theorem may be the most significant achievement in the field after the improvement of the Routh-Hurwitz criterion. The essential success of this theorem is that it enables us to decide if a linear time-invariant control system with several uncertain parameters stay stable as the parameters change over a set. The theorem provides an exact answer, that is, nonconservatively, when the parameters arise linearly or multilinearly in characteristic polynomials. This solves some significant control system design problems such as 1) the calculation of the real parametric stability margin, 2) the determination of stability margins under mixed parametric uncertainty, 3) the evaluation of the worst case or robust performance measured in the $\mathrm{H}_{\infty}$ norm over a prescribed parameter uncertainty set, and 4) the extension of classical design techniques involving Nyquist, Nichols, and Bode plots and root-loci to systems containing several uncertain real parameters.

*Corresponding Author: ugurozdemir@anadolu.edu.tr

Received: 13 March 2018 Accepted: 22.10.2018 
In the real world, most systems are nonlinear. A linear time-invariant model is acquired by positioning the operating point and linearizing the system equation about it. As the operating point varies so do the parameters. Therefore there is significant uncertainty with respect to the real plant. Kharitonov's Theorem caused an immense revival of work on robust stability under real parametric uncertainty. Scientists started to believe that the robust control problem for parametric uncertainties could be treated without conservatism and overbounding. Furthermore, it has shown the effectiveness and transparency of methods that use the algebraic and geometric characteristics of the stability region in parameter space by contrasting the blind formulation of an optimization problem. This has resulted in an outpouring of results in this field over the last few years [1].

Edge Theorem extended Kharitonov's Theorem by considering dependencies between the coefficients of the polynomial and addressing the general stability region. It provides a complete, exact, and constructive characterization of the root set of a polytopic family.

Edge Theorem proves that the root region of the entire family can be attained from the root set of the edges. Because the edges are one-parameter sets of polynomials, this theorem effectively and constructively decreases work load of determining the root space under multiple-parameter uncertainty in a set of one-parameter root locus problems.

Kharitonov's Theorem was first published in 1978 [2]. Bialas [3] and Barmish [4] are recognized for presenting it to Western literature. Several applications of this theorem are accessible in articles by Bose [5], Yeung and Wang [6], Minnichelli, Anagnost, and Desoer [7], and Chapellat and Bhattacharyya [8].

The dynamic characteristics of aircraft longitudinal motion and its control are studied by linearizing aircraft equations of motion $[9,10]$. There are typically two modes in the longitudinal dynamics of an aircraft: 1) short period and 2) long period (Phugoid). The differences in the dynamic properties of aircraft longitudinal motion result from the differences in geometry and mass properties of the aircraft expressed as stability derivatives. Stability derivatives differ for different aircraft and may also differ for the same aircraft under different flight conditions. Hence any stability analysis performed for a certain flight condition is only valid for that condition.

In this study, a longitudinal stability analysis of an unmanned aerial vehicle (UAV) is performed for two uncertain stability derivatives in all flight conditions. This study includes the following steps: the geometry and mass properties of the UAV are given. The stability and control derivatives of the UAV are calculated using AAA (Advanced Aircraft Analysis) software [11-12]. A stability analysis of the UAV's longitudinal motion is performed for a specific flight condition (an operation point). Finally, two stability derivatives, which change considerably for different flight conditions, are determined to be the uncertain parameters.

The first part of the paper introduces the theory and methods behind robust stability analysis approaches, such as gridding techniques and the Edge and Bialas [14-17] theorems. Next, the application of these methods to the longitudinal aircraft motion under the uncertainty of two stability derivatives is demonstrated. In this new method, aircraft longitudinal stability analysis for all flight conditions is reduced to four polynomials obtained with the Edge and Bialas theorems. An exact answer of whether the aircraft is stable or not for all values in a given range is obtained from the eigenvalues of four matrices of the four polynomials. The stability analysis of the UAV under parametric uncertainties is repeated for three uncertainty ranges.

\section{THE UAV AND ITS DERIVATIVES}

This section introduces the UAV used for stability analysis. Its geometric configuration and the modeled flight condition determine the values of the stability derivatives. These stability derivatives form the magnitude of the coefficients in the transfer functions. 
First, the stability and control derivatives are obtained AAA software [11] for certain flight conditions. It is expected that the values of stability derivatives in the whole flight envelope vary around these values and within a bounded range. Some derivatives have more uncertain behavior with wider ranges, and some have less. Second, two stability derivatives, which have a wide range, are selected as the uncertain parameters. Three different uncertainty levels, $20 \%, 40 \%$, and $50 \%$, are used as limits for the stability derivatives, and these bounds are taken into account in the stability analyses.

\subsection{The Geometry of the UAV}

The geometry of the fixed-wing UAV [12] and flight conditions were modeled in the AAA program (Figs. 1-2).

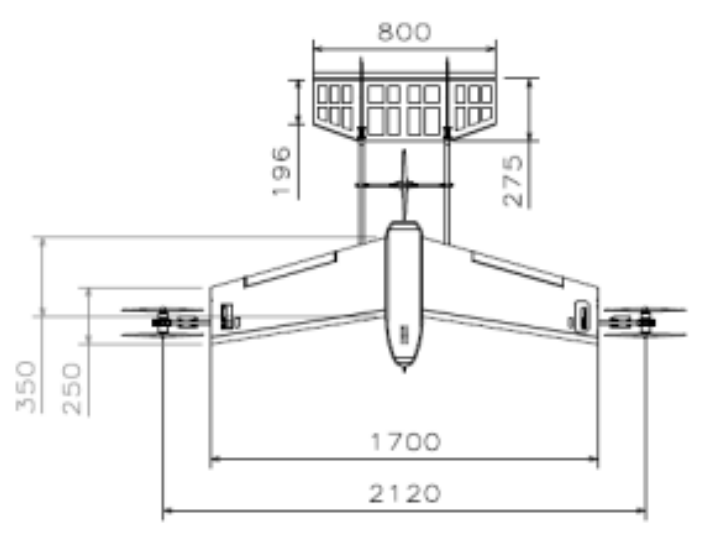

Figure 1. Top View of the UAV (dimensions in $\mathrm{mm}$ )

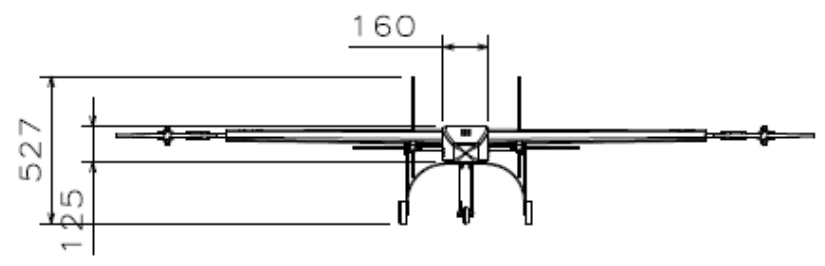

(a)

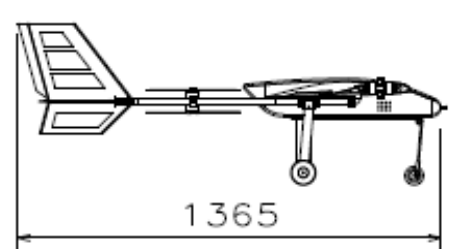

(b)

Figure 2. (a) Fron View of the UAV; (b) Side View of the UAV (dimensions in mm)

The UAV's weight and velocity information are presented in Table 1.

Table 1. Weights and velocity information

\begin{tabular}{ll}
\hline Parameter & Aircraft \\
\hline$W_{e}, \mathrm{~N}$ & 57.761 \\
\hline$W_{\text {Prp }}, \mathrm{N}$ & 26.478 \\
\hline$W_{p l}, \mathrm{~N}$ & 12.033 \\
\hline$W_{0}, \mathrm{~N}$ & 96.272 \\
\hline$V_{\text {cr }}, \mathrm{m} / \mathrm{s}$ & 21 \\
\hline$V_{\text {stall }}, \mathrm{m} / \mathrm{s}$ & 16 \\
\hline
\end{tabular}




\subsection{Stability and Control Derivatives of the UAV}

The stability and control derivatives of the UAV were calculated for a velocity of $20 \mathrm{~m} / \mathrm{s}$ at an altitude of 842 masl [12]. The derivatives required for longitudinal motion are given in Table 2.

Table 2. Stability and control derivatives of the UAV

\begin{tabular}{|c|c|c|c|c|c|}
\hline$C_{L_{0}}$ & 0.6311 & $C_{D_{0}}$ & 0.0660 & $C_{m_{0}}$ & -0.2327 \\
\hline$C_{L_{u}}$ & 0.0020 & $C_{D_{u}}$ & 0 & $C_{m_{u}}$ & 0.0006 \\
\hline$C_{L_{\alpha}}$ & $5.2472 \mathrm{rad}^{-1}$ & $C_{D_{\alpha}}$ & $0.3241 \mathrm{rad}^{-1}$ & $C_{m_{\alpha}}$ & $-0.8101 \mathrm{rad}^{-1}$ \\
\hline$C_{L_{\dot{\alpha}}}$ & $3.2213 \mathrm{rad}^{-1}$ & $C_{D_{\dot{\alpha}}}$ & 0 & $C_{m_{\dot{\alpha}}}$ & $-7.5846 \mathrm{rad}^{-1}$ \\
\hline$C_{L_{q}}$ & $6.4108 \mathrm{rad}^{-1}$ & $C_{T_{u}}$ & -0.0660 & $C_{m_{q}}$ & $-18.2663 \mathrm{rad}^{-1}$ \\
\hline$C_{L_{\delta e}}$ & $0.0601 \mathrm{rad}^{-1}$ & $C_{z_{\alpha}}$ & $-\left(C_{L_{\alpha}}+C_{D_{0}}\right)$ & $C_{m_{\delta e}}$ & $-1.4876 \mathrm{rad}^{-1}$ \\
\hline$C_{L_{i h}}$ & $1.3706 \mathrm{rad}^{-1}$ & $C_{Z_{\dot{\alpha}}}$ & $-2 C_{L_{h \alpha}} \eta V h \frac{d \varepsilon_{h}}{d \alpha}$ & $C_{m_{i h}}$ & $-3.6583 \mathrm{rad}^{-1}$ \\
\hline$C_{L_{h \alpha}}$ & $3.1597 \mathrm{rad}^{-1}$ & $C_{Z_{q}}$ & $-2 C_{L_{h \alpha}} \eta V h$ & $M_{\mathrm{u}}$ & $0.0008 \mathrm{~m}^{-1} \cdot \mathrm{s}^{-1}$ \\
\hline$X_{\mathbf{u}}$ & $-0.0800 \mathrm{~s}^{-1}$ & $C_{Z_{\delta e}}$ & $-C_{L_{\delta e}}$ & $M_{T_{u}}$ & 0.0000 \\
\hline $\boldsymbol{X}_{T_{u}}$ & 0.0000 & $Z_{\mathrm{u}}$ & $-0.7662 \mathrm{~s}^{-1}$ & $M_{w}$ & $-1.1565 \mathrm{~m}^{-1} \cdot \mathrm{s}^{-1}$ \\
\hline$X_{w}$ & $0.18607 \mathrm{~s}^{-1}$ & $Z_{w}$ & $-3.2203 \mathrm{~s}^{-1}$ & $M_{\dot{w}}$ & $-0.0812 \mathrm{~s}^{-1}$ \\
\hline$X_{\delta e}$ & 0 & $Z_{\dot{w}}$ & -0.0104 & $M_{q}$ & $-3.9115 \mathrm{~s}^{-1}$ \\
\hline$X_{\alpha}$ & $3.7214 \mathrm{~m} / \mathrm{s}^{-2}$ & $Z_{q}$ & $-0.6319 \mathrm{~m} / \mathrm{s}$ & $M_{\delta e}$ & $-42.473 \mathrm{~s}^{-2}$ \\
\hline$Z_{\alpha}$ & $-64.406 \mathrm{~m} / \mathrm{s}^{-2}$ & $Z_{\delta e}$ & $-1.4285 \mathrm{~m} / \mathrm{s}^{2}$ & $M_{\alpha}$ & $-23.13 \mathrm{~s}^{-2}$ \\
\hline$Z_{\dot{\alpha}}$ & $-0.208 \mathrm{~m} / \mathrm{s}^{-1}$ & & & $M_{\dot{\alpha}}$ & $-1.624 \mathrm{~s}^{-1}$ \\
\hline
\end{tabular}

\section{THE LINEARIZED UAV MODEL AND STABILITY ANALYSIS}

The linearized dynamic model of a fixed-wing aircraft can be represented by Eq. 1 [11]:

$$
\left[\begin{array}{ccc}
\left(s-X_{u}-X_{T_{u}}\right) & -X_{\alpha} & g \cos \theta_{1} \\
-Z_{u} & \left\{s\left(U_{1}-Z_{\dot{\alpha}}\right)-Z_{\alpha}\right\} & \left\{-\left(Z_{q}+U_{1}\right) s-g \sin \theta_{1}\right\} \\
-\left(M_{u}+M_{T_{u}}\right) & -\left\{M_{\dot{\alpha}} s+M_{\alpha}+M_{T_{\alpha}}\right\} & \left(s^{2}-M_{q} s\right)
\end{array}\right]\left[\begin{array}{c}
\frac{u(s)}{\delta_{e}(s)} \\
\frac{\alpha(s)}{\delta_{e}(s)} \\
\frac{\theta(s)}{\delta_{e}(s)}
\end{array}\right]=\left[\begin{array}{c}
X_{\dot{\delta}} \\
Z_{\dot{\delta}} \\
M_{\dot{\delta}}
\end{array}\right]
$$

Three transfer functions corresponding to the elevator inputs can be found with Cramer's Rule. For example, the transfer function for velocity change in $\mathrm{x}$-direction with the change of the elevator input can be shown as follows (Eq 2): 


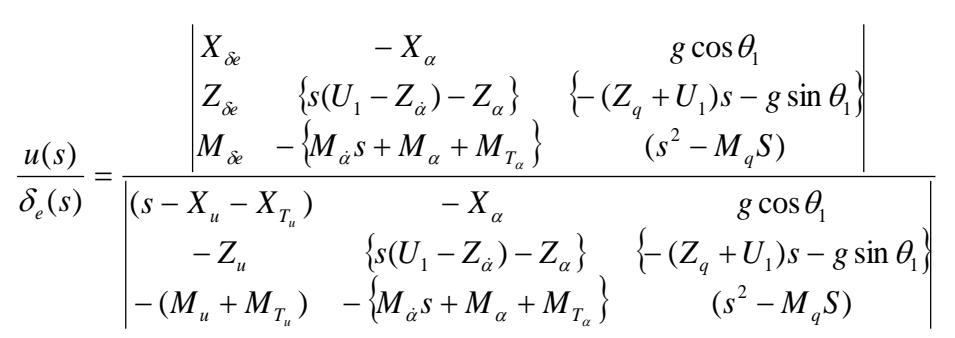

The stability and control derivatives given in Table II are substituted in Eq. 2 and the transfer function is obtained (Eq. 3).

$$
\frac{u(s)}{\delta_{e}(s)}=\frac{-5.31 s^{2}+5568 s+26549}{21 s^{4}+182 . s^{3}+739 s^{2}+73 s+173}
$$

The characteristic equation is the denominator of the transfer function (Eq. 4):

$$
21 s^{4}+182 . s^{3}+739 s^{2}+73 s+173=0
$$

The stability of the UAV around an operation point can be decided by looking at the location of the roots of the characteristic equation in the complex plane (Eqs. 5, 6).

$$
\begin{gathered}
\left.S_{1,2}=-4.3 \pm 4 i \quad \text { Short Period }\right) \\
S_{2,3}=-0.02 \pm 0.5 i \quad(\text { Phugoid Mode })
\end{gathered}
$$

Since all roots are at the left-hand side of the complex plane as seen in Figure 3, the UAV is stable for this operation point (flight condition: $20 \mathrm{~m} / \mathrm{s}$ and 842 masl ).

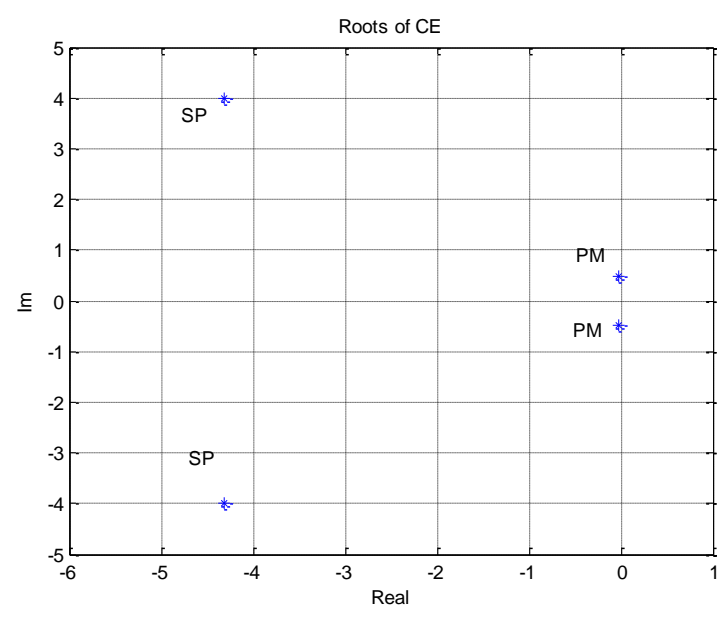

Figure 3. Roots of the Characteristic Equation

Figure 3 shows that the Phugoid Mode roots are quite close to the origin. Therefore, the Phugoid mode is critical for stability and dominant for longitudinal motion. From the damping ratio $\xi$ and the natural frequency $\omega_{n}$, the time required to halve the amplitude is calculated from Eq. 3. Table 3 presents the UAV's longitudinal modes.

$$
T_{1 / 2_{p m}}=\frac{0.693}{\xi \omega_{n}}
$$


Table 3. Longitudinal modes of the UAV

\begin{tabular}{c|c} 
Phugoid Mode & Short Period Mode \\
\hline$\xi_{p m}=0.0417$ & $\xi_{s p}=0.7313$ \\
$\omega_{n_{p m}}=0.48 \frac{\mathrm{rad}}{\mathrm{s}}$ & $\omega_{n_{s p}}=5.88 \frac{\mathrm{rad}}{\mathrm{s}}$ \\
$T_{1 / 2_{p m}}=34 \mathrm{~s}$ & $T_{1 / 2_{s p}}=0.1612 \mathrm{~s}$
\end{tabular}

The UAV has dampened responses to elevator inputs. However, in the Phugoid Mode, the dampening ratio is very low, indicating that even small changes in the stability derivatives may result in instability.

\section{THE UNCERTAINTY OF STABILITY DERIVATIVES}

The UAV operates in a flight envelope with different stability derivatives under different flight conditions. Normally the stability analysis has to be repeated for all different possible flight conditions, but since there are infinite possible flight conditions, this is not feasible. Experience shows [5] that uncertainty in flight derivatives usually falls in the range of $20-50 \%$. In this study, uncertainty for the two stability derivatives $X_{\alpha}$ and $Z_{\alpha}$ were tested at $20 \%, 40 \%$, and $50 \%$ (Table 4). For example, test 1 allows both stability derivatives to vary by $20 \%$ greater or less the nominal values, which are based on the operation point described in section 2.1.

Table 4. Three Different Uncertainty Levels

\begin{tabular}{ll} 
Case 1: & $0.8 X_{\alpha}<X_{\alpha}<1.2 X_{\alpha}$ \\
& $0.8 Z_{\alpha}<Z_{\alpha}<1.2 Z_{\alpha}$ \\
\hline Case 2: & $0.6 X_{\alpha}<X_{\alpha}<1.4 X_{\alpha}$ \\
& $0.6 Z_{\alpha}<Z_{\alpha}<1.4 Z_{\alpha}$ \\
\hline Case 3: & $0.5 X_{\alpha}<X_{\alpha}<1.5 X_{\alpha}$ \\
& $0.5 Z_{\alpha}<Z_{\alpha}<1.5 Z_{\alpha}$
\end{tabular}

\section{ROBUST STABILITY ANALYSIS METHODS}

This section introduces the theory behind the robust stability analysis. Stability analysis is expanded from a single operation point to include the entire flight envelope based on the uncertainty of two stability derivatives.

\subsection{Gridding}

By gridding the uncertain parameters in Fig. 4, pole spreading (Fig. 5) can be performed by marking the poles for every point in Fig. 4. In the example, all poles are on the left-hand side, so stability is assured for the given range of parametric uncertainty. Pole spreading methods require intense calculation, especially when there are many uncertain parameters. Moreover, no matter how small the gridding is, stability is not theoretically assured since this method only addresses a sample of all possible conditions [14]. 


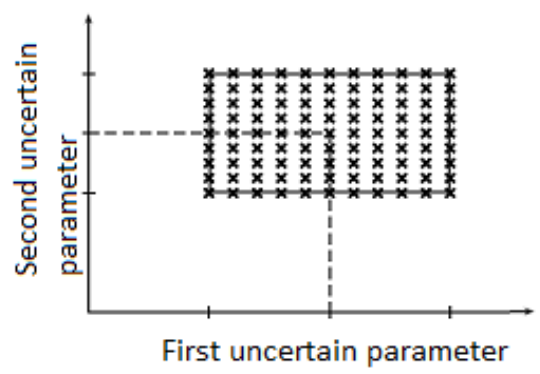

Figure 4. Gridding of two uncertain parameters

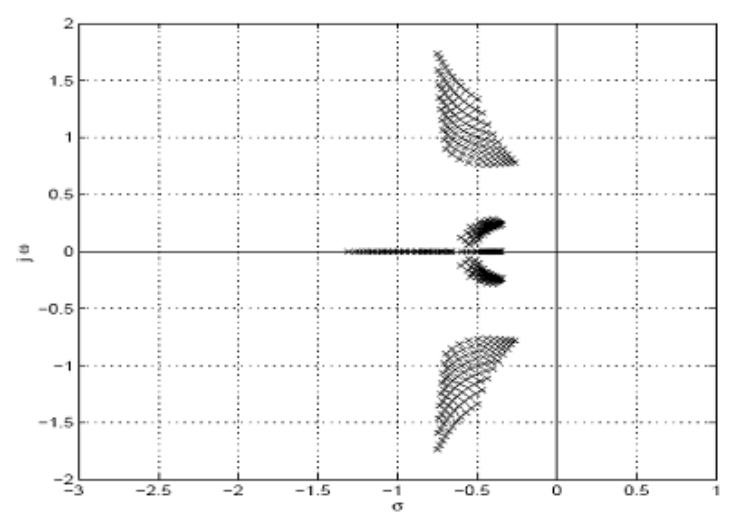

Figure 5. Root set for the grid points in Figure 4

\subsection{Affine-linear Coefficient Polynomials and Value Sets}

The characteristic equation of an aircraft's longitudinal motion with two uncertain parameters is a polynomial with affine linear coefficients. This section discusses this kind of polynomials and the Edge theorem $[14,15]$ for stability analysis. Polynomials with affine-linear coefficients can be presented as follows:

$$
P(s, \mathbf{q})=P_{0}(s)+q_{1} P_{1}(s)+\ldots \ldots .+q_{l} P_{l}(s)
$$

where:

$\mathbf{q}=\left[\begin{array}{lllll}q_{0} & q_{1} & \ldots \ldots \ldots & q_{l}\end{array}\right]^{T}$

$q_{i} \in\left[\begin{array}{ll}q_{i}^{-} & q_{i}^{+}\end{array}\right] \quad i=1,23 \ldots . l$

$q_{0} \quad q_{1} \quad \ldots \ldots . . \quad q_{l}:$ uncertain parameters

$q_{i}^{-}$and $q_{i}^{+}:$lower and upper boundary of the $\mathrm{i}^{\text {th }}$ uncertain parameter

For example;

$$
\begin{aligned}
& P(s, \mathbf{q})=s^{4}+q_{0}\left(s^{3}+s^{2}-s\right)+q_{1}\left(s^{2}+s\right)+q_{2}\left(3 s^{2}+1\right) \\
& q_{0}, q_{1}, q_{2} \in\left[\begin{array}{ll}
0 & 1
\end{array}\right]
\end{aligned}
$$

is a polynomial with the affine linear coefficients.

The value set of a polynomial family with three uncertain parameters can be seen in Fig.6. The value set of the polynomial family for all frequency values must not include the origin for stability. 


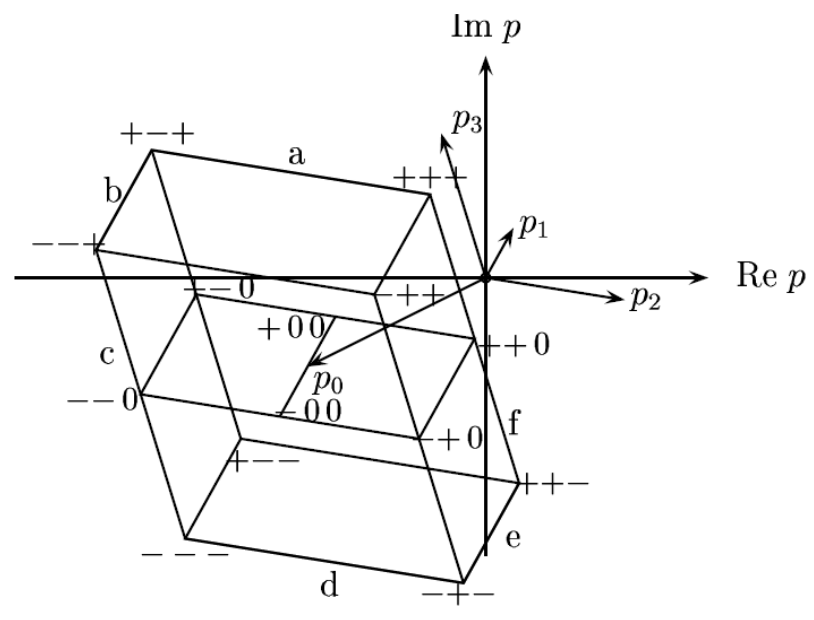

Figure 6. The construction of the value set with three parameters and fixed frequency [15]

\subsection{The Edge Theorem}

The Edge theorem simplifies stability analysis because it shows evaluating only polynomials is sufficient to evaluate stability. The theorem states that the polynomial family

$$
P(s, Q)=\left\{P(s, \mathbf{q})=\sum_{i=0}^{n} a_{i}(\mathbf{q}) s^{i} \mid \quad \mathbf{q} \in Q\right\}
$$

with affine coefficient functions $a_{i}(\mathbf{q})$ and

$Q=\left\{\mathbf{q} \mid \quad q_{i} \in\left[q_{i}^{-}, q_{i}^{+}\right] i=1,2, \ldots, l\right\}$

is stable if and only if the edges of $Q$ are stable.

For a given polynomial family, the polynomials of a parameter box's vertices are called vertex polynomials. At these vertex polynomials, the uncertain parameters are at their minimum and maximum values. The edge polynomial can be obtained from two vertex polynomials as follows:

$$
P(s, Q)=\left\{(1-\lambda) P_{b}(s)+\lambda P_{c}(s) \mid \lambda \in[0 ; 1]\right\}
$$

where

$P_{b}(s): 1^{\text {st }}$ vertex polynomial

$P_{c}(s): 2^{\text {nd }}$ vertex polynomial

$\lambda$ : uncertain parameter in the edge polynomial

Note that no matter how many uncertain parameters there are, once the vertex polynomials are obtained with the minimum and maximum values of the uncertain parameters, the edge polynomial includes only one uncertain parameter, as seen in Eq. 10. Next, we use the Bialas theorem [16, 17], which can evaluate polynomial stability with one uncertain parameter. 


\subsection{Bialas Theorem}

Let $H_{n}^{b}$ and $H_{n}^{c}$ be the Hurwitz matrices of

$P_{b}(s)=b_{o}+b_{1} s+b_{2} s^{2}+\ldots \ldots \ldots . .+b_{n} s^{n}, b_{n}>0$

and

$P_{c}(s)=c_{o}+c_{1} s+c_{2} s^{2}+\ldots \ldots \ldots+c_{n} s^{n}, c_{n}>0$

respectively [6].

$P(s, Q)=\left\{(1-q) P_{b}(s)+q P_{c}(s) \quad \mid q \in[0 ; 1]\right\}$

is stable if and only if

1) $P_{b}(s)$ is stable.

2) the matrix $\left(H_{n}^{b}\right)^{-1} H_{n}^{c}$ has no non-positive real eigenvalues.

The Bialas theorem thus makes it possible to easily evaluate the stability of the edge polynomials with one uncertain parameter.

\section{Application of Robust Analysis Methods to the Longitudinal Stability Analysis of the UAV}

The characteristic equation for the aircraft longitudinal motion can be expressed as follows:

$$
C E=\left|\begin{array}{ccc}
\left(s-X_{u}-X_{T_{u}}\right) & -X_{\alpha} & g \cos \theta_{1} \\
-Z_{u} & \left\{s\left(U_{1}-Z_{\dot{\alpha}}\right)-Z_{\alpha}\right\} & \left\{-\left(Z_{q}+U_{1}\right) s-g \sin \theta_{1}\right\} \\
-\left(M_{u}+M_{T_{u}}\right) & -\left\{M_{\dot{\alpha}} s+M_{\alpha}+M_{T_{\alpha}}\right\} & \left(s^{2}-M_{q} s\right)
\end{array}\right|
$$

If the values of the stability derivative except $X_{\alpha}$ and $Z_{\alpha}$ are substituted to Eq. 11, the CE has the following form (Eq. 12):

$$
\begin{aligned}
C E= & 173.116+42.0954 s+479.841 s^{2}+117.73 s^{3} \\
& +21.208 s^{4}+0.000273752 X_{\alpha}+2.9807 s X_{\alpha} \\
& +0.7662 s^{2} X_{\alpha}-0.00784322 Z_{\alpha}-0.31292 s Z_{\alpha} \\
& -3.9915 s^{2} Z_{\alpha}-s^{3} Z_{\alpha}
\end{aligned}
$$

The CE is a polynomial family with affine linear coefficients hence

$C E=a_{0}+a_{1} s+a_{2} s^{2}+a_{3} s^{3}+a_{4} s^{4}$

where

$$
\begin{aligned}
& a_{0}=173.116+0.000273752 X_{\alpha}-0.00784322 Z_{\alpha} \\
& a_{1}=42.0954+2.9807 X_{\alpha}-0 . .31292 Z_{\alpha} \\
& a_{2}=479.841+0.7662 X_{\alpha}-3.9915 Z_{\alpha} \\
& a_{3}=117.73-Z_{\alpha} \\
& a_{4}=21.208
\end{aligned}
$$

For case 1, uncertainties are in the ranges of $0.8 X_{\alpha}<X_{\alpha}<1.2 X_{\alpha}$ and $0.8 Z_{\alpha}<Z_{\alpha}<1.2 Z_{\alpha}$. when the numerical values of the stability derivatives are substituted:

$$
2.977<X_{\alpha}<4.465 \quad \text { and } \quad-77.287<Z_{\alpha}<-51.524
$$


The minimum and/or maximum values of the uncertain parameters were substituted in Eq. 12 and four vertex polynomials were obtained (Eqs. 13-16).

$$
\begin{aligned}
& P^{--}(s)=173.521+67.0924 s+791.754 s^{2}+195.017 s^{3}+21.208 s^{4} \\
& P^{-+}(s)=173.521+79.591 s+791.754 s^{2}+169.255 s^{3}+21.208 s^{4} \\
& P^{++}(s)=173.521+79.591 s+687.783 s^{2}+169.255 s^{3}+21.208 s^{4} \\
& P^{+-}(s)=173.521+67.0924 s+687.783 s^{2}+195.017 s^{3}+21.208 s^{4}
\end{aligned}
$$

Vertex polynomials are required to obtain edge polynomials, which can be expressed as follows:

$$
\begin{aligned}
& \text { edge } 1=(1-\lambda) P^{--}(s)+\lambda P^{+-}(s) \\
& \text { edge } 2=(1-\lambda) P^{+-}(s)+\lambda P^{++}(s) \\
& \text { edge } 3=(1-\lambda) P^{++}(s)+\lambda P^{-+}(s) \\
& \text { edge } 4=(1-\lambda) P^{-+}(s)+\lambda P^{--}(s)
\end{aligned}
$$

If Eqs. 13-16 are substituted in Eqs. 17-20 the following edge polynomials are obtained:

Edge polynomial 1:

$$
\begin{aligned}
& (1-\lambda)\left(173.521+67.0924 s+791.754 s^{2}+195.017 s^{3}+21.208 s^{4}\right) \\
& +\lambda \quad\left(173.521+67.0924 s+687.783 s^{2}+195.017 s^{3}+21.208 s^{4}\right)
\end{aligned}
$$

Edge polynomial 2:

$$
\begin{aligned}
& (1-\lambda)\left(173.521+67.0924 s+687.783 s^{2}+195.017 s^{3}+21.208 s^{4}\right) \\
& +\lambda \quad\left(173.521+79.591 s+687.783 s^{2}+169.255 s^{3}+21.208 s^{4}\right)
\end{aligned}
$$

Edge polynomial 3:

$$
\begin{aligned}
& (1-\lambda)\left(173.521+79.591 s+687.783 s^{2}+169.255 s^{3}+21.208 s^{4}\right) \\
& +\lambda \quad\left(173.521+79.591 s+791.754 s^{2}+169.255 s^{3}+21.208 s^{4}\right)
\end{aligned}
$$

Edge polynomial 4:

$$
\begin{array}{lr}
(1-\lambda) & \left(173.521+79.591 s+791.754 s^{2}+169.255 s^{3}+21.208 s^{4}\right) \\
+\lambda & \left(173.521+67.0924 s+791.754 s^{2}+195.017 s^{3}+21.208 s^{4}\right)
\end{array}
$$

As seen in Eqs. 21-24, the edge polynomials include one uncertain parameter. The Bialas theorem is very useful because it provides a very effective and simple means of checking the stability of polynomials with a single uncertain parameter. If all four edge polynomials are stable, then it can be said that aircraft longitudinal motion is stable for the given parametric uncertainty. This procedure is repeated for three test cases with different ranges (Table 5). 
Özdemir et al. / Eskişehir Technical Univ. J. of Sci. and Tech. A - Appl. Sci. and Eng. 19(4)-2018

Table 5. Stability analysis for $0.8 X_{\alpha}<X_{\alpha}<1.2 X_{\alpha}$ and $0.8 Z_{\alpha}<Z_{\alpha}<1.2 Z_{\alpha}$

\begin{tabular}{|c|c|c|c|}
\hline & Hurwitz Determinants of $\mathbf{P}_{\mathbf{b}}(\mathbf{s})$ & $\begin{array}{c}\text { Eigenvalues of the } \\
\text { Matrix }\left(\mathbf{H}_{\mathbf{n}}^{\mathbf{b}}\right)^{-\mathbf{1}} \mathbf{H}_{\mathbf{n}}{ }^{\mathbf{c}}\end{array}$ & \\
\hline Edge 1 & $195.017,152983 ., 3.66469 \times 10^{6}$ & $1 ., 1 ., 0.626687$ & Stable \\
\hline Edge 2 & $195.017,132707 ., 2.29661 \times 10^{6}$ & $2.09246,1 ., 0.864456$ & Stable \\
\hline Edge 3 & $169.255,114723 ., 4.15419 \times 10^{6}$ & $1.33855,1 ., 1$. & Stable \\
\hline Edge 4 & $169.255,132320 ., 5.5606 \times 10^{6}$ & $1.15618,1 ., 0.57002$ & Stable \\
\hline
\end{tabular}

Table 6. Stability analysis for $0.6 X_{\alpha}<X_{\alpha}<1.4 X_{\alpha}$ and $0.6 Z_{\alpha}<Z_{\alpha}<1.4 Z_{\alpha}$

\begin{tabular}{|c|c|c|c|}
\hline & Hurwitz Determinants of $\mathbf{P}_{\mathbf{b}}(\mathbf{s})$ & $\begin{array}{c}\text { Eigenvalues of the } \\
\text { Matrix }\left(\mathbf{H}_{\mathbf{n}}^{\mathbf{b}}\right)^{-\mathbf{1}} \mathbf{H}_{\mathbf{n}}^{\mathbf{c}}\end{array}$ & \\
\hline Edge 1 & $207.898,174122 ., 3.09861 \times 10^{6}$ & $1 ., 1 ., 0.145488$ & Stable \\
\hline Edge 2 & $207.898,130891 ., 450810$. & $2.09246,1 ., 0.864456$ & Stable \\
\hline Edge 3 & $156.374,97601.5,4.12765 \times 10^{6}$ & $\begin{array}{l}1.67863,1 .+/ \text { - i } \\
3.83297 \times 10^{-14}\end{array}$ & Stable \\
\hline Edge 4 & $156.374,130118 ., 6.92879 \times 10^{6}$ & $1.33823,1 ., 0.334179$ & Stable \\
\hline
\end{tabular}

Table 7. Stability analysis for $0.5 X_{\alpha}<X_{\alpha}<1.5 X_{\alpha}$ and $0.5 Z_{\alpha}<Z_{\alpha}<1.5 Z_{\alpha}$

\begin{tabular}{|c|c|c|c|}
\hline & Hurwitz Determinants of $\mathbf{P}_{\mathbf{b}}(\mathbf{s})$ & $\begin{array}{c}\text { Eigenvalues of the } \\
\text { Matrix }\left(\mathbf{H}_{\mathbf{n}}{ }^{\mathbf{b}}\right)^{-1} \mathbf{H}_{\mathbf{n}}{ }^{\mathbf{c}}\end{array}$ & \\
\hline Edge 1 & $214.339,185194 ., 2.72431 \times 10^{6}$ & $1 ., 1 .,-0.18889$ & Unstable \\
\hline Edge 2 & $214.339,129481 .,-514594$. & $-11.4029,1 ., 0.691473$ & Unstable \\
\hline Edge 3 & $149.933,89543.1,4.05749 \times 10^{6}$ & $1.8573,1 ., 1$. & Stable \\
\hline Edge 4 & $149.933,128515 ., 7.53598 \times 10^{6}$ & $1.44109,1 ., 0.250858$ & Stable \\
\hline
\end{tabular}

\section{CONCLUSION}

UAV linearized longitudinal motion was investigated for a fixed flight condition. Since stability derivatives may have different values for different flight conditions, two stability derivatives were selected as uncertain parameters. Various bounded ranges $(20 \%, 40 \%$, and $50 \%)$ were accounted for by these uncertain parameters. The theory and methods behind the robust stability analysis were briefly presented. The longitudinal stability analysis of the UAV for all flight conditions was reduced to the analysis of four polynomials with one uncertain parameter. The stability analysis of these four polynomials was performed with the Bialas theorem, which is very useful for polynomials with only one uncertain parameter. Thus, instead of making a very large number of calculations for every possible flight condition, a stability analysis was simplified to four polynomials. For test cases 1 and 2 (20\% and 40\% uncertainty ranges), the aircraft is stable under all flight conditions. However, in test case 3 (50\% uncertainty range), the aircraft was not stable under all conditions. This study has shown this effectiveness of this approach with two uncertain parameters. Future work may include additional uncertain parameters. 


\section{ACKNOWLEDGEMENTS}

This research was supported as a Scientific Research Project by Anadolu University (Project 1705F288).

\section{REFERENCES}

[1] Bhattacharyya, S.P., Chapellat, H., and Keel, L.H., Robust Control: The Parametric Approach, Prentice Hall PTR, Upper Saddle River, NJ, 1995.

[2] Kharitonov, V. L., Asymptotic stability of an equilibrium position of a family of systems of linear differential equations, Differential Uravnen, vol. 14, pp.2086-2088, 1978.

[3] Bialas, S., A necessary and sufficient condition for the stability of interval matrices, International Journal of Control, vol. 37, pp. 717 - 722, 1983.

[4] Barmish, B.R., Invariance of strict Hurwitz property of polynomial concept for robust stability problems with linearly dependent coefficient perturbations, IEEE Transactions on Automatic Control, vol. AC-29, no. 10, pp. 935-936, 1984.

[5] Bose, N.K., A system-theoric approach to stability of sets of polynomials, Contemporary Mathematics, vol. 47, pp. 25-34, 1985.

[6] Yeung, K. S, and Wang, S. S., A simple proof of Kharitonov's theorem, IEEE Transaction on Automatic Control, vol. 32, no.4, pp. 822-823, 1987.

[7] Minnichelli, R. J., Anagnost, J. J., and Desoer, C. A., An elementary proof of Kharitonov's stability theorem with extensions, IEEE Transactions on Automatic Control, vol. AC-34, no.9, pp. 995-998, 1989.

[8] Chapellat, H. and Bhattacharyya, S. P., An alternative proof of Kharitonov's theorem, IEEE Transactions on Automatic Control, vol. AC-34, no.4, pp. 448-450, 1989.

[9] Yechout T.R., Morris S.L., Bossert D.E., and Hallgren W.F. Introduction To Aircraft Flight Mechanics: Performance, Static Stability, Dynamic Stability, And Classical Feedback, AIAA, 2003.

[10] Blakelock J.H., Automatic Control of Aircraft and Missiles, 1st edition, John Wiley \& Sons, New York, 1965.

[11] Roskam J, Airplane Flight Dynamics, And Automatic Controls, DARcorporation, USA, 2003.

[12] Oznalbant Z., Kavsaoglu M. S., and Cavcar M., Design, Flight Mechanics and Flight Demonstration of a Tiltable Propeller VTOL UAV", presented at 16th AIAA Aviation Technology, Integration and Operations Conference, AIAA Aviation Forum, 2016.

[13] Sadraey M. H., Design of a Nonlinear Robust Controller for a Complete Unmanned Aerial Vehicle Mission, Ph.D. Thesis, University of Kansas, 1995.

[14] Soylemez M. T., Pole Assignment for Uncertain Systems, Research Studies Press (RSP), Baldock, UK, 1999.

[15] Ackerman J., Robust Control: The Parameter Space Approach, Springer, New York, London, 2002. 
Özdemir et al. / Eskişehir Technical Univ. J. of Sci. and Tech. A - Appl. Sci. and Eng. 19(4)-2018

[16] Bialas S., A Necessary And Sufficient Condition For The Stability Of Convex Combinations Of Stable Polynomials Or Matrices, Bull. Polish Academy of Sciences, vol. 33, no. 9-10, pp.473-480, 1988.

[17] Bialas S., A Necessary And Sufficient Condition For The Stability Of Convex Combinations Of Stable Polynomials Or Matrices, Bull. Polish Academy of Sciences, vol. 33, no. 9-10, pp.473-480, 1988. 